\section{Working with GFP in the Brain}

BioTechniques 34:492-494 (March 2003)

A powerful technique currently used widely to track cells in the central nervous system is that of enhanced GFP as a fluorescent marker. Cells can be infected with a GFP-expressing retrovirus $(1,2)$ or taken from GFP transgenic animals and transplanted into a syngeneic host (3). However, this approach may be confounded by considerable autofluorescence within the mammalian central nervous system. It is difficult to distinguish cells labeled with GFP from those that are naturally fluorescent. In the past, the problem with autofluorescence has been so severe that researchers have used nonfluorescent immunohistochemistry with an anti-GFP antibody to track the marked cells (4). This approach solves the problems associated with background fluorescence due to lipofuscin but negates the enormous strength of endogenous fluorescence of GFP to track cell migration and localization. In addition, the potential to co-localize GFP with additional fluorescent markers is no longer possible with such strategies. Our goal here is to discuss a strategy for recognizing when green fluorescence in the central nervous system is due to GFP and not the result of a naturally occurring fluorescent molecule.

The cause for most of the naturally occurring fluorescence in the central nervous system is lipofuscin. Lipofuscin is a complex of peroxidized lipids, proteins, and transition metals (5) and is the result of the incomplete breakdown of macromolecules within the lysosomal system. Undegraded material undergoes peroxidation and polymerization to form a heterogeneous complex. Oxidative enzymes catalyze the peroxidation with an increased deposition in cells that are undergoing higher levels of oxidative metabolism. Lipofuscin makes fluorescence microscopy in the central nervous system difficult because it has a very broad excitation and emission spectrum. We have recorded lipofuscin fluorescing in 4',6-diamidino-2-phenylindole (DAPI), fluorescein isothiocyanate (FITC), rhodamine, and far red channels. This problem is compounded by the fact that lipofuscin also has a very widespread distribution. One study that looked at the topographical distribution of lipofuscin in lemurs found the pigment in the brain stem, neocortex, cerebellum, hypothalamus, basal forebrain, hippocampus, and olfactory bulb (5). In our study with 20 -week-old mice, we have found that the mitral cells of the olfactory bulb have the highest depostion of lipofuscin granules, an observation consistent with the high level of oxidative metabolism of these cells (5).

Wide-field epifluorescent microscopy offers numerous advantages for visualization of GFP-labeled cells. Greater numbers of tissue sections can be observed in less time and at less cost than with a confocal microscope. A caveat, however, is that GFP and lipofuscin fluorescence is particularly difficult to distinguish when using a widefield microscope. Green fluorescent cells observed using a FITC filter set (excitation 490/20 nm, emission 528/38 $\mathrm{nm})$ are frequently a mixture of lipofuscin-containing cells and GFP-containing cells (Figure 1A). One way to differentiate GFP-containing cells from lipofuscin-containing cells is to use higher magnifications when it is some-
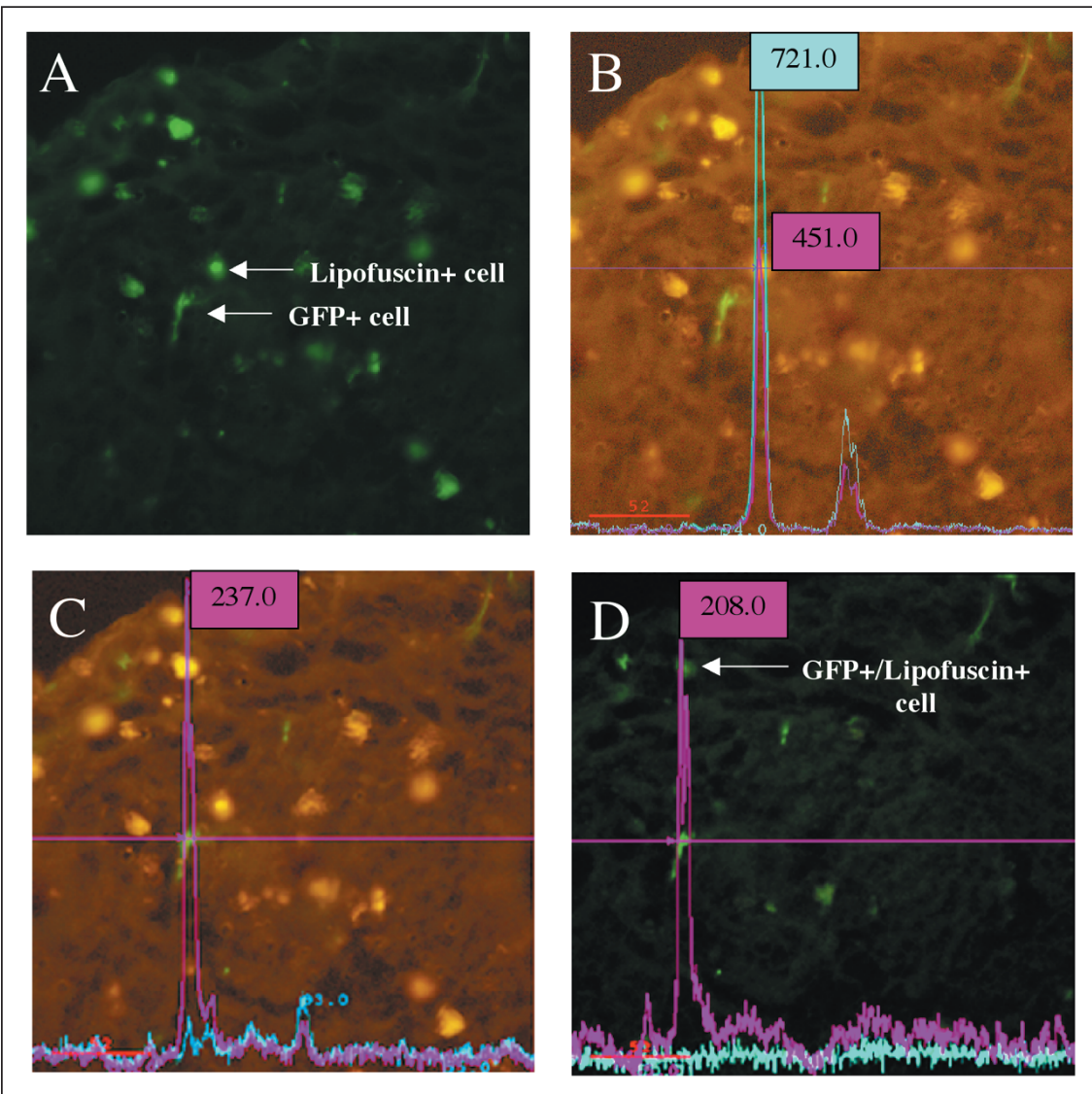

Figure 1. Sudan Black B removal of lipofuscin fluorescence. (A) Green fluorescence present in the olfactory bulb of a mouse that has received bone marrow cells from a GFP transgenic donor eight weeks earlier, as seen with a wide-field epifluorescent microscope using a FITC filter. Green fluorescence is due to both GFP and lipofuscin. Arrows indicate a GFP-positive (GFP+) and a lipofuscin-positive (lipofuscin+) cell. (B) Combined image from different excitation wavelengths to show the total fluorescence emitted from each cell (obtained using a deconvolution microscope). By comparing A and B, it is evident that only one cell in panel A is fluorescing solely within the GFP wavelengths. This demonstrates the difficulty of recognizing when cells fluoresce due to GFP using a wide-field epifluorescent microscope for detection. The relative intensity of fluorescence of a lipofuscin-positive cell and a GFP-positive cell are shown in panels B and C, respectively. The blue line represents red fluorescence, while the red line represents green fluorescence. Note that lipofuscin is emitting even more green light than GFP (451 compared to 237). (D) Sudan Black B effectively eliminates the lipofuscin fluorescence while only marginally reducing the intensity of the GFP fluorescence (237 compared to 208). The treatment reveals that one of the lipofuscin-positive cells also contains GFP (arrow). Scale in micrometers. 
times possible to tell the two apart by looking at the distribution of green fluorescence within each cell. Lipofuscin is stored within lysosomes and gives cells a distinct granular fluorescence (Figure 2A), while GFP is a soluble protein that gives cells a more even green fluorescence. Low magnification, which is often used to quantify and obtain spatial information of fluorescent cells, does not allow the aforementioned morphologic distinctions necessary to distinguish GFP from lipofuscin. One is required to determine which cells fluoresce in the FITC channel only compared with those that fluoresce in all channels (lipofuscin positive). This approach is cumbersome and does not resolve the difficulty of detecting cells in the central nervous system that contain both GFP and lipofuscin, which can be a significant problem in regions that are rich in lipofuscin deposits.
There are other problems that may be encountered when using a widefield epifluorescent microscope to detect GFP-containing cells in the centra nervous system. Occasionally, GFP fluorescence is detected in other channels, depending on the effectiveness of the filters being used. In addition, cells that have accumulated large amounts of lipofuscin may have a fluorescence that appears more even than granular. Therefore, we recommend two additional tests to ensure that green fluorescence is due to GFP and not the result of lipofuscin. We recommend first the use of a deconvolution or confocal microscope with narrow band filters to characterize the emission spectrum from a representative sample of cells (Figure 1, B and C). It is not always correct to assume that fluorescence at the correct wavelength is due to GFP. Assessment of fluorescence at other wavelengths is critical to be sure that light of any other wavelength is not emitted. This is the most reliable means to discriminate between lipofuscin and GFP fluorescence because their emission spectra differ markedly. Lipofuscin emits light over a broad spectrum (430-670 nm), while GFP emits light in much a narrower spectrum (480-525 $\mathrm{nm}$ ) (Figure 1, B and C) (6). It should also be noted that, while we found most of the light emitted by lipofuscin to appear in the rhodamine channel (excitation $555 / 28 \mathrm{~nm}$, emission $617 / 73 \mathrm{~nm}$ ) of a wide-field microscope, it still often emitted more green light than GFP (Figure 1, B and C).

The use of a confocal or deconvolution microscope to analyze the emission spectrum of a green fluorescent cell is a dependable way to determine whether fluorescence is due to GFP. However, for added certainty, we recommend the 


\section{Benchmarks}
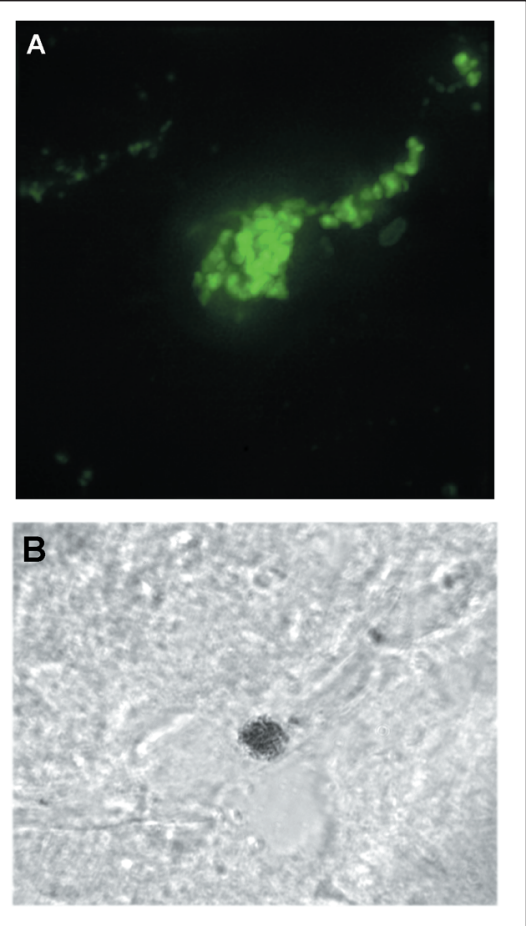

Figure 2. High-magnification view of a lipofuscin-containing cell (A) and example GFP immunohistochemistry (B). (A) High-magnification view of a lipofuscin-containing cell from the olfactory bulb of a 20 -week-old mouse. Note the distinctive punctate appearance of lipofuscin accumulation in storage granules. (B) A cell stained with an anti-GFP antibody. This confirms that the fluorescence seen after Sudan Black B treatment is due to GFP and not an alternative autofluorescent central nervous system molecule. Primary antibody: GFP monoclonal antibody (1:500; BD Biosciences Clontech, Palo Alto, CA, USA). Secondary antibody: Anti-mouse IgG-HRP (1:500).

use of an anti-GFP antibody to identify GFP positive cells by immunohistochemical detection (Figure 2B). Immunohistochemistry on a sample of fluorescent cells circumvents problems arising from other sources of autofluorescence in the central nervous system, such as the ubiquitous redox carrier flavin, which has an emission spectrum of 520-560 nm, quite similar to GFP.

We wish to emphasize that the problem of strong background fluorescence in the central nervous system in general, and with lipofuscin specifically, is not new. However, with the emergence of GFP methods to track cells in the central nervous system, this problem is more widely encountered, although not completely recognized. One simple method used in the past that seems to be over- looked by current researchers is that of Sudan Black quenching $(7,8)$. This method entails the treatment of tissue sections with $0.3 \%$ Sudan Black B in $70 \%$ ethanol. We found that incubation of tissue sections for $10 \mathrm{~min}$ followed by eight rinses in PBS successfully eliminates lipofuscin fluorescence without effects on GFP fluorescence (Figure 1D). This technique has the added advantage that it enables the identification of cells that contain both GFP and lipofuscin, as lipofuscin fluorescence no longer masks GFP fluorescence.

In summary, as GFP becomes increasingly popular as a means of tracking cells in the central nervous system, it is important to realize that not all green fluorescence is due to GFP. Hence, we suggest that a characterization of the emission spectra of a representative sample of the identified cells be performed that can be confirmed by immunohistochemistry with an antiGFP antibody. We recommend treatment with Sudan Black B to eliminate autofluorescence, particularly in cases where quantification or co-localization of GFP using additional fluorescent markers is desired.

\section{REFERENCES}

1.Van Praag, H., A.F. Schinder, B.R. Christie, N. Toni, T.D. Palmer, and F.H. Gage. 2002. Functional neurogenesis in the adult hippocampus. Nature 415:1030-1034.

2.Priller, J., A. Flugel, T. Wehner, M. Boentert, C.A. Haas, M. Prinz, F. FernandezKlett, K. Prass, et al. 2001. Targeting genemodified hematopoietic cells to the central nervous system: use of green fluorescent protein uncovers microglial engraftment. Nat. Med. 7:1356-1361.

3.Brazelton, T.R., F.M.V. Rossi, G.I. Keshet, and H.M. Blau. 2000. From marrow to brain: expression of neuronal phenotypes in adult mice. Science 290:1775-1779.

4.Zhao, L., W. Duan, M. Reyes, C.D. Keene, C.M. Verfaille, and W.C. Low. 2002. Human bone marrow stem cells exhibit neurological deficits after grafting into the ischemic brain of rats. Exp. Neurol. 174:11-20.

5.Gilissen, E.P., R.E. Jacobs, E.R. McGuinness, and J.M. Allman. 1999. Topographical localization of lipofuscin pigment in the brain of the aged fat-tailed dwarf lemur (Cheirogaleus medius) and grey lesser mouse lemur (Microcebus murinus): comparison to iron localization. Am. J. Primatol. 49:183193.

6.Billinton, N. and A.W. Knight. 2001. Seeing the wood through the trees: a review of techniques for distinguishing GFP from endoge- nous autofluorescence. Anal. Biochem. 291:175-197.

7.Romijn, H.J., J.F.M. Van Uum, I. Breedijk, J. Emmering, I. Radu, and C.W. Pool. 1999. Double immunolabeling of neuropeptides as analysed by confocal laser scanning fluorescence microscopy. J. Histochem. Cytochem. 47:229-235.

8.Schnell, S.A., W.A. Staines, and M.W. Wessendorf. 1999. Reduction of lipofuscinlike autofluorescence in fluorescently labeled tissue. J. Histochem. Cytochem. 47:719-730.

This work is supported by National Institutes of Health grant nos. NS39492 (M.S.-P.) and NS24728 (R.P.S.). Address correspondence to Kristian P. Doyle, Department of Molecular Microbiology and Immunology, Oregon Health and Science University, 3181 Sam Jackson Park Road, Portland, OR 97239, USA. e-mail: doylekr @ohsu.edu

Received 13 December 2002; accepted 23 January 2003.

\section{Kristian P. Doyle, Roger P. Simon ${ }^{1}$, Aurelie Snyder, and Mary P. Stenzel-Poore Oregon Health and Science University \\ ${ }^{1}$ Robert S. Dow Neurobiology Laboratories \\ Portland, OR, USA}

